\title{
Concepts Are Not a Natural Kind*
}

\author{
Edouard Machery†‡
}

$†$ To contact the author, please write to Edouard Machery, Department of History and Philosophy of Science, University of Pittsburgh, Pittsburgh, PA 15260; e-mail: machery@pitt.edu.

$\ddagger$ I would like to thank the anonymous referees for their very useful comments. 


\begin{abstract}
In cognitive psychology, concepts are those data structures that are stored in long-term memory and are used by default in human beings' higher cognitive processes (categorization, inductive and deductive reasoning...). Most psychologists of concepts assume that these mental representations share many scientifically important properties, and the psychology of concepts is expected to describe those properties. Psychologists assume thereby that concepts constitute a natural kind. I call this assumption the Natural Kind Assumption. This article challenges the Natural Kind Assumption. It is argued that a growing body of evidence suggests that concepts do not constitute a natural kind. Hence, the notion of concept is inappropriate to carve human beings' mental representations at their joints, if one aims at formulating scientifically relevant inductive generalizations about the human mind.
\end{abstract}




\section{Introduction}

In cognitive science, concepts are the data structures that are stored in long-term memory and are used by default in the higher cognitive processes (categorization, inductive and deductive reasoning, analogy making, language understanding....). ${ }^{1}$ Cognitive scientists assume typically that they constitute a homogenous class of mental representations. Of course, everybody recognizes that there are plenty of differences between concepts and also between kinds of concepts: DOG and CAT have different properties and so do folk biological concepts and concepts of artifacts. But it is assumed that over and above these differences, the mental representations that are used in our higher cognitive processes share a large number of properties. The goal of a psychological theory of concepts is to capture those properties. Since it is assumed that their properties explain many properties of the higher cognitive processes, developing an adequate theory of concepts is a prerequisite for a good cognitive theory.

Such a view is nicely put by the psychologist Gregory Murphy in his recent survey of the psychology of concepts:

The psychology of concepts cannot by itself provide a full explanation of the concepts of all the different domains that psychologists are interested in. This book will not explore the psychology of concepts of persons, musical forms, numbers, physical motions, and political systems. The details of each of these must be discovered by the specific disciplines that study them (...). Nonetheless, the general processes of concept learning and representation may well be found in each of these domains. For example, I would be quite surprised if concepts of musical 
forms did not follow a prototype structure (chapter 2), did not have a preferred level of categorization (chapter 7), and did not show differences depending on expertise or knowledge (chapter 6). (2002, 2-3, my emphasis)

This view of concepts says essentially that concepts constitute a natural kind, that is, a class of entities about which many inductive generalizations can be formulated. I call the view that concepts constitute a natural kind the natural kind assumption or, for short, the NK assumption.

The NK assumption is widely accepted (sometimes explicitly, e.g., Medin et al. 2000, 123; Prinz 2002; Goldstone and Kersten 2003, 599) by psychologists, philosophers of psychology and, more generally, cognitive scientists. Arguably, it has been part and parcel of the psychology of concepts. Psychologists believe that with the notion of concept, they have identified one of the important classes for a scientific psychology - one of those classes that support the formulation of many inductive generalizations. For more than thirty years (Machery ms), they have been looking for generalizations about members of this class. ${ }^{2}$ In effect, they have been assuming the NK assumption.

Despite the widespread endorsement of the NK assumption, this paper sets out to challenge it. Indeed, I make a case for the following claim:

A growing amount of evidence suggests that concepts do not constitute a natural kind.

I am claiming that, contrary to what has been assumed in cognitive science for thirty years, the data structures that are used by default in our higher cognitive processes do not constitute a homogenous kind about which many inductive generalizations can be 
formulated. Thus, the notion of concept is not an adequate way of carving human beings' mental representations at their joints, if one aims at formulating scientifically relevant inductive generalizations about the human mind.

Before going any further, I should discard a possible misunderstanding. In this paper, I am not addressing the usual questions raised about concepts by philosophers of mind. I take for granted the notion of concept that prevails in cognitive psychology - data structures that are used by default in our higher cognitive processes. The issue is then the following: psychologists believe that the class of concepts is important for a scientific psychology, because it supports many inductive generalizations. This is the NK assumption: concepts are a natural kind. In this paper, I set out to reject this assumption.

In the first section, I cast some light on the NK assumption, spelling out what properties the class of concepts should possess in order to be a natural kind. On this basis, I present in the second section the argument from explanatory necessity, which shows that the class of concepts does not possess these properties. It claims that psychologists have to posit several kinds of concepts that have little in common in order to explain our higher cognitive processes. In the last two sections, I defend two premises of this argument. In the third section, I show that the main psychological theories of concepts have posited three theoretical entities that have little in common. In the last section, I present some empirical evidence, drawn from the psychological research on concept combination, that we are justified in positing all these entities. I conclude that the NK assumption is misconceived.

\section{The Natural Kind Assumption}




\subsection{Natural Kind and Induction}

The notion of natural kind is essentially enmeshed with the problem of induction (Mill 1905; Quine 1969; Boyd 1990, 1991; Hacking 1991). For, the notion of natural kind singles out those classes about which non accidental, scientifically relevant inductive generalizations can be formulated. It assumes that there are two kinds of classes: those about which inductive generalizations can be formulated, e.g., atoms, and those about which no or few generalizations can be formulated, e.g., things that weigh more than 124 kilos. Thus, the members of a natural kind are supposed to share non accidentally a large number of (logically unrelated), scientifically important properties (or relations) beside the properties (or relations) that are used to identify them.

Since members of natural kinds have many properties in common, natural kinds are the building blocks of scientific generalizations. Many empirical sciences aim at identifying the natural kinds in their domain in order to develop adequate empirical theories. Psychology is no exception. Psychologists look for classes of entities about which scientifically relevant generalizations could be made. They introduce new notions to pick out kinds that are believed to be natural (e.g., the notion of basic emotion) and they reject notions that turn out to pick out kinds that are not natural (Griffiths 1997).

\subsection{Two Red Herrings: Essences and Laws}

Now this core idea has been cashed out in many ways. Which account is best suited for my present purpose? Clearly, this account has to be both applicable to psychological kinds and to be broad. Otherwise, the denial of the NK assumption would simply be trivial. 
Two accounts are thus inadequate. According to a common account, natural kinds are characterized by the possession of an essence, that is, a set of intrinsic, causally explanatory properties that are necessary and jointly sufficient to belong to the kind (Putnam 1975). However, it is unlikely that psychological kinds possess essences - at least, if the functionalist view is true. And this notion is excessively restrictive: for example, species wouldn't be natural kinds according to this account (Hull 1978). One might want to broaden the notion of essence, by including relational properties alongside intrinsic ones. However, this notion of natural kind would remain unsatisfactory. For, it does not distinguish the conditions of membership in the kind from the possession of causally explanatory properties. However, in some natural kinds, the properties that are causally explanatory are not necessary conditions of membership, for not all members possess these properties. The class of political parties in democratic societies illustrates this point. The conditions of membership in this class are arguably different from the causal properties that explain why most democratic political parties share many properties.

According to another account, natural kind terms feature in laws, that is, in generalizations that are temporally and spatially unrestricted and that support counterfactuals (Collier 1996). Now, psychological kinds feature in ceteris paribus generalizations - not in laws (Fodor 1974). And, again, this notion is too restrictive: few kind terms feature in laws. One could propose to replace the notion of law by the notion of ceteris paribus generalization. This is a step in the right direction, as we shall see below. However, an important element is missing from this account, namely the idea that there is 
at least one causal mechanism that accounts for these generalizations. This is an important aspect of the kinds scientists are interested in for inductive purposes.

\subsection{The Causal Notion of Natural Kind}

A broader notion has been developed by Richard Boyd (1990, 1991; Griffiths 1997).

A class $C$ of entities is a natural kind if and only if there is a large set of scientifically relevant properties such that $\mathrm{C}$ is the maximal class whose members tend to share these properties because of some causal mechanism.

The core idea of this definition is the following. A natural kind is a class about which many generalizations can be formulated: its members tend to have many properties in common. These generalizations are not accidental: there is at least one causal mechanism that explains why its members tend to have those properties. Finally, this class is not a subset of a larger class about which the same generalizations could be formulated.

One might object that "large” is too vague. However, this complaint is unfounded. For the notion of natural kind, like the notion of heap, is vague. Even if there is no way to sharpen the idea of "a large set of properties", it remains that the number of properties that can be projected from one subset of the class to the whole class is one dimension that distinguishes natural kinds from other classes.

Inversely, one might object that a class of entities that would share only few scientifically important properties would be a natural kind. First, one can question whether such classes really exist. These fundamental properties would plausibly cause the members of this class to share other properties. Moreover, if there were such classes, I would stick to 
my guns: they would not be natural kinds. For, they would be noticeably different from the paradigmatic natural kinds. Instead of stretching the notion of natural kind in order to include them, it would arguably be better to distinguish two types of scientific kinds: the natural kinds whose members share many properties and, say, the fundamental kinds, whose members share few fundamental properties.

In the present context, two aspects of the definition are important. First, the properties that characterize a natural kind are not necessarily possessed by all its members. It is only required that members of a natural kind tend to have these properties. Hence, natural kind terms don't have to feature in laws. It is only required that they feature in ceteris paribus generalizations.

Second, essences are only one of the possible causal mechanisms that explain why members of a natural kind share or tend to share many properties. Boyd has insisted upon the homeostasis of properties (1990): in some natural kinds, the occurrence of a property causes the occurrence of other properties and is caused by their occurrence. Other mechanisms are possible, including common descent and social causes (Griffiths 1997).

\subsection{The NK Assumption}

The causal notion of natural kind is relevant for my present purpose. It allows psychological kinds to be natural kinds, since natural kinds do not have to possess essences nor to feature in laws. Moreover, this notion has a large extension: substances, e.g., gold, physical entities, e.g., atoms, species, e.g., dogs, and artifacts do qualify as natural kinds. However, the causal notion of natural kind is not vacuous. It implies that nominal kinds, 
for instance, the class of physical objects that weigh more than 30 kilos, are not natural kinds. For their members don’t share many (scientifically relevant) properties. Moreover, it implies that many subsets of natural kinds, e.g., white dogs, are not natural kinds either. For the properties that are true of white dogs are true of all dogs (except being white).

In what follows, I use exclusively the causal notion of natural kind. Hence, the NK assumption says that the class of data structures that are used by default in our higher cognitive processes possesses three properties:

- $\quad$ There is a large set $S$ of properties that these data structures tend to possess.

- They possess these properties because of some causal mechanism.

- This set of properties is specific to this class of data structures.

Importantly, psychologists who endorse the NK assumption are not committed to deny the existence of differences between concepts or kinds of concepts (Medin et al. 2000). For despite these differences, concepts may share a large set of properties.

\subsection{The Received View}

For more than thirty years, the cognitive psychology of concepts has been built on the view that concepts constitute a homogenous class about which specific, scientifically relevant generalizations can be formulated. Although some psychologists have recently endorsed the heterogeneity hypothesis (e.g., Ashby et al. 1998; Hahn and Chater 1998; Smith et al. 1998), many still endorse this view, particularly prototype theorists (e.g., Hampton 2001; Smith 2002), exemplar theorists (e.g., Juslin and Persson 2002; Zaki and Nosofky 2004), and theory theorists (e.g., Carey 2000). Barsalou and colleagues assume that most 
concepts, if not all, are perceptual symbols (Barsalou 1999). The recent search for hybrid models of concepts (see Section 5.6) suggests similarly that psychologists have not renounced the belief that concepts constitute a homogeneous class of representations. Several philosophers of psychology share this commitment. For instance, Prinz (2002) proposes that most concepts, if not all, are proxytypes.

Moreover, the received view has driven the methodology of the psychology of concepts and of the higher cognitive functions. In substance, psychologists have tried to find data that were easily accounted for by their pet theory of concepts, but that were problematic for rival theories. Rival theories could account for these data only with ad hoc assumptions. Now, this methodology assumes that the class of concepts is homogenous and, therefore, that all the data about concepts have to be accounted for by a unique theory. This is tantamount to assume the NK assumption.

\section{The Argument From Explanatory Necessity}

\subsection{The Heterogeneity Hypothesis}

Given the causal notion of natural kind, there are three options to rebut the NK assumption:

- It could not be the case that the data structures that are used by default in the higher cognitive processes have many properties in common.

- These mental representations could have many properties in common by accident.

- $\quad$ They could have those properties because they are a subset of a larger set of representations: no empirical generalization could be made specifically about them. 
I propose that the available empirical evidence about our concepts supports strongly the first option: it is not the case that concepts have in common a large set of properties. Indeed, I will argue that the class of concepts is divided into several kinds of representations that have little in common. I call this claim the heterogeneity hypothesis. There are several readings of this hypothesis. One could claim that different types of categories, say the folk biological categories and the artifact categories, are represented by different kinds of concepts and that these kinds have little in common. For example, there could be few properties common to DOG and TABLE, because in general, concepts of biological kinds and concepts of artifacts could have little in common. One could also claim that different cognitive processes are defined over different kinds of concepts and that these kinds have little in common. For example, a concept of dogs used in the categorization process could have little in common with a concept of dogs used in induction, because in general, concepts that are used in the categorization process and concepts that are used in the induction process could have little in common. I reject both readings.

Instead, I claim that most categories are represented by several concepts that belong to kinds that have little in common. For example, we have several concepts of dogs, each of which belongs to a different kind of concepts. There are few properties common to these concepts because in general, the corresponding kinds of concepts have little in common. I call these kinds "the basic kinds of concepts". Each of these concepts of dogs can be used in our various higher cognitive processes. We can use $\mathrm{DOG}_{1}, \mathrm{DOG}_{2}, \mathrm{DOG}_{3}$ etc. to categorize, to reason inductively, to reason deductively etc. They qualify as concepts 
because they are poised to be used in our higher cognitive processes. They are data structures that are used by default in cognitive processes. Safe for specific circumstances, we rely on these data structures, when we decide whether something is a dog, when we reason about dogs... Beliefs about dogs that are not part of these data structures can be used in our cognitive processes as well. However, they are not poised to be used in them. They are retrieved from long-term memory merely occasionally. This idea is summarized by Figure 1.

Put Figure 1 about here.

This view of our higher cognition stands in sharp contrast with the received view. Our higher cognitive processes are not defined over a homogenous class of mental representations. Thus, the class of concepts is not useful to draw scientifically relevant generalizations about our mental representations. Instead of trying unproductively to characterize the properties that are generally possessed by concepts, psychologists and other cognitive scientists' explicit goal should be to characterize each basic kind of concepts. I submit that the properties that are characteristic of each of them are not characteristic of the others. This is the right grain for theorizing about our mental representations.

Some might object that we knew all along that we use different kinds of knowledge in our higher cognitive processes. Isn't it obvious that we can use any kind of knowledge to decide whether something is a dog? Granted. But this is beside the point. For the claim 
is (i) that we have specific data structures in our mind that are poised for being used in our cognitive processes and (ii) that these structures divide into different kinds that have little in common. That we may use any kind of information in our higher cognitive processes does not say anything about the class of data structures that are used by default in our higher cognitive processes. Psychologists and some philosophers of psychology aim at formulating generalizations about them, assuming thereby that they are homogeneous. I deny this claim.

\subsection{The Argument From Explanatory Necessity}

It is now possible to state the following argument against the NK assumption:

\section{The Argument from Explanatory Necessity}

1. Concepts, that is, the data structures used by default in our higher cognitive processes, are posited in order to explain the properties of these processes.

2. The theoretical constructs posited by the main theories of concepts have little in common.

3. In order to explain the properties of our higher cognitive processes, we need to assume that these theoretical constructs are used by default in our higher cognitive processes.

4. Hence, our higher cognitive processes use several kinds of representations that have little in common.

5. Hence, concepts are not a natural kind. 
The core idea of this argument is straightforward. Concepts are theoretical entities that are posited in order to explain the properties of our higher cognitive processes. In order to provide a satisfactory explanation, we have to posit several kinds of concepts that have little in common. Which ones? At least those that have been assumed by the main paradigms of concepts in the recent psychological literature.

Clearly, this argument is empirical. The truth of premise 3 is a matter of fact. Thus, the whole argument claims that the available empirical evidence about concepts and about the higher cognitive processes supports the denial of the NK assumption. Since this argument is empirical, new empirical data could result in a different conclusion.

I will take premise 1 for granted. Almost all psychologists and many philosophers of psychology (e.g., Prinz 2002) assume it. Concepts are those data structures that we use by default in our higher cognitive processes. Their properties explain how we reason, how we categorize etc. In what follows, I argue for premises 2 and 3.

\section{Three Kinds of Representations that Have Little in Common}

In this section, I defend the second premise of the argument from explanatory necessity. I first present the three main paradigms of concepts in the contemporary literature on concepts. Then, I show that they posit three theoretical constructs that have little in common.

\subsection{Three Theoretical Entities: Prototypes, Exemplars, Theories}


Since the rejection of the classical view of concepts in the 1970's, cognitive psychology has produced three main views, the prototype view of concepts, the exemplar view of concepts, and the theory view of concepts. ${ }^{3}$

The prototype view of concepts is built around the idea that concepts are prototypes (e.g., Rosch and Mervis 1975; Smith et al. 1988; Smith and Minda 2002)ㄹ:

A concept of a class of objects is a prototype, i.e., a data structure that encodes some statistical information about the properties possessed by the members of this class, particularly, about the properties possessed by most members of this class. Prototypes single out the properties of the members of the denoted category in statistical terms. In most prototype models (but not in all), prototypes are assumed to encode some information about the typical properties of the denoted category, that is, the properties that are judged to be possessed by most members of the category. Importantly, these properties are not judged to be necessary. For example, to have a concept of dogs is to think by default of dogs as having generally, but not necessarily, such form, as having generally a tail etc. ${ }^{5}$

According to the exemplar view of concepts, our higher cognitive processes are defined over representations of specific category members, which stand for the whole category (e.g., Medin and Shaffer 1978; Nosofsky 1988; Juslin and Persson 2002).

A concept of a class of objects consists of $a$ set of exemplars, i.e., of representations of particular members of this class. ${ }^{6}$ 
For example, to have a concept of dogs is to think by default of dogs as being the class of entities that are similar to Fido, Pluto, and Spot and to use these individual representations to reason about dogs in general.

According to the theory view of concepts, our mental representations are similar to scientific theories and the cognitive processes are similar to scientific forms of reasoning (Murphy and Medin 1985; Carey 1985; Keil 1989; Gopnik and Meltzoff 1997; Keil and Wilson 2000). ${ }^{7}$

A concept of a class of entities is a theory about this class, that is, a data structure that encodes some nomological, causal, modal and functional generalizations about this class

To possess a concept of dogs is to think by default of dogs as being the class of entities that satisfy some kind of theory, i.e., some nomological, modal, causal and functional generalizations (e.g., a theoretical concept of dogs may say of dogs that they necessarily engender individuals that belong to the same species).

\subsection{Prototypes, Exemplars and Theories have Little in Common}

The claim is that these three theoretical constructs have little in common. Now, how does one evaluate how different various kinds of mental representations are? Clearly, exemplars, theories and prototypes have plenty of irrelevant properties in common, for example not being cups of tea. What matters, however, are those properties that psychologists take to be important when they aim at characterizing mental representations. Psychologists focus on the representational properties of mental representations and on their functional properties, i.e., what mental representations represent and how they are used in cognitive processes. 
Hence, if exemplars, prototypes and theories have different representational and functional properties, this would suggest that they have little in common. ${ }^{8}$

$>$ Prototypes, exemplars and theories are assumed to encode three different types of knowledge

- According to exemplar theorists, applying a concept to an individual is tantamount to think that this individual is similar to some specific individuals, which are themselves described as having such and such properties. Thus, the mind stores some information about the properties of specific individuals, for example, some descriptions of Fido and Spot.

- According to prototype theorists ${ }^{9}$, applying a concept to an individual is tantamount to think that this individual possesses to a sufficient degree a sufficient number of typical properties. Thus, the mind stores some statistical information about the properties that characterize classes of individuals.

- According to theory theorists, applying a concept to an individual is tantamount to think that this individual possesses some properties either with some modal force, or because of some law, or for some causal or functional reason. Thus, the mind is storing some modal, nomological, causal and functional information about classes of individuals. For most categories, it is possible to encode some information about the typical properties of their members, some modal, nomological and causal information about them and some information about the properties possessed by some of them. Hence, most categories could be represented by a prototype, a theory and a set of exemplars. 
Prototypes, exemplars and theories are assumed to be used in three different kinds of cognitive processes $^{10}$

- Exemplar theorists and prototype theorists suppose that the higher cognitive processes involve the computation of the similarity between some representations and, respectively, sets of exemplars and prototypes.

To make this a bit more concrete, consider how a prototype theorist might explain the categorization process. Peter sees a dog. He forms a representation that attributes some properties to the dog. The properties that are attributed are matched with the properties that are represented by several prototypes, including the prototype of dogs. The similarity between the dog and each prototype is computed. Since the similarity between the dog and the prototype of dogs is higher than the similarity between the dog and the other prototypes, Peter concludes that he is seeing a dog. Various properties of our other higher cognitive processes are explained in similar terms (see Osherson et al. 1990 on induction; see Hampton 1982, 1988 and Sloman 1998 on deduction; see Osherson and Smith 1989 on probabilistic reasoning).

Advocates of the exemplar view would provide a similar explanation of the categorization process, except that the dog would be compared to sets of exemplars, instead of prototypes (Nosofsky 1988). Many properties of the other higher cognitive processes could be explained in the same terms (e.g., Juslin and Persson 2002).

- Advocates of the exemplar view and of the prototype view assume different ways of computing the similarity between a represented object and, respectively, sets of exemplars 
and prototypes. In brief, prototype theorists assume that the similarity measure is a linear function of the number of properties common to the concept and the object to be categorized, while exemplar theorists take it to be a non linear function.

- The theory view of concepts assumes that our higher cognitive processes do not rely on similarity. On the contrary, they are supposed to be similar to the reasoning processes that are used in science. Since theory theorists are less concerned with our cognitive processes than prototype and exemplar theorists, their views on our cognitive processes are less developed. Theory theorists often compare our reasoning processes to explanations (Keil and Wilson 2000) or to inferences to the best explanation (Murphy and Medin 1985). They generally assume that our cognitive processes follow the standard rules of logic and of probabilistic reasoning (Smith et al. 1992).

Murphy and Medin (1985) provide the following toy example. If at a party, a guest jumps in the swimming pool entirely dressed, we may conclude that she is drunk. This categorization does not rest on any matching between our concept of drunken people and our singular representation of this individual. On the contrary, we infer that this is the most plausible explanation of the behavior of this individual. This inference is supposed to follow the rules of probabilistic inference.

The first point shows that prototypes, exemplars and theories are assumed to possess different types of representational properties. The second one shows that they are assumed to possess different types of functional properties. Hence, given the properties that are relevant to characterize mental representations, I conclude that the main 
psychological theories of concepts assume three kinds of mental representations that have little in common. This supports premise 2. In other words, I have established the following conditional claim: if it can be shown that these three kinds of data structures are used by default in our higher cognitive processes, then concepts are not a natural kind.

\section{Concept Combination and the NK Assumption}

I propose that we are justified in positing the three kinds of representations described above. It is of course impossible to review in this article the large body of evidence that supports this claim. In this section, I focus instead on a specific cognitive competence, the capacity to combine concepts. Since Osherson and Smith’s seminal study (1981), concept combination has been a hot topic among psychologists of concepts (see the reviews in Hampton 1997 and Murphy 2002, chap. 12). Their results suggest that concept combination requires the kinds of knowledge that exemplars, prototypes and theories are assumed to represent. This is evidence for premise 3 of the argument from explanatory necessity. ${ }^{11}$

\subsection{Concept Combination}

Concept combination is the capacity to form new data structures about classes for which we have no data structure permanently stored in long-term memory. Those representations, e.g., GRANDMOTHER SPY, are formed on the basis of other representations stored in long-term memory, e.g., GRANDMOTHER and SPY. A concept is simple if and only if it is a permanent data structure in long-term memory. There is a finite stock of simple concepts. A complex concept is not stored permanently in long-term memory, but has to be 
produced on the fly for various cognitive purposes (e.g., for language comprehension, reasoning etc.). The claim that concepts combine means that there is a mental mechanism that operates on simple concepts to produce complex concepts (or on less complex concepts to produce more complex concepts). ${ }^{12}$

To understand concept combination is to characterize the processes that underlie the creation of a new data structure out of the data structures that are stored in long term memory. As a result, we would explain why some information turns out to be represented by complex concepts. The claim defended here is that in order to explain concept combination, one has to assume the storage of prototypes, exemplars and theories in longterm memory.

\subsection{Prototypes and Property Inheritance}

When subjects are given two expressions, say “Oxford graduate students” and "carpenter", ${ }^{13}$ and when they are asked to list the properties that are typical of Oxford graduate students who are carpenters, they often find the task meaningful and easy (Hampton 1987; Kunda et al. 1990; Johnson and Keil 2000). In some experiments, they are also asked to determine which properties are typical of the two original categories, in our example, Oxford graduate students and carpenters (Hampton 1987; Johnson and Keil 2000). The psychologist James Hampton has shown that there is a correlation between the properties that are judged to be typical of the members of the original categories, Oxford graduate students and of carpenters, and the properties that are judged to be typical of the 
members of the resulting category, Oxford graduate students who are carpenters (Hampton 1987).

Now, prototypes are supposed to encode this very type of knowledge. This suggests that the creation of a complex concept involves determining which properties are typical of the denoted category, Oxford graduate students who are carpenters, on the basis of the prototypes of the original categories, Oxford graduate students and carpenters. When we produce on the fly a complex concept, we retrieve those prototypes and we transmit some of the properties they represent to the complex concept. ${ }^{14}$ This is known as property inheritance (Hampton 1997). The inheritance of properties by the complex concept is $\left(\right.$ partly ${ }^{15}$ ) driven by the assumption that the typical properties of the categories denoted by the compounded concepts are also typical of the category denoted by the complex concept. Property inheritance is summarized by Figure 2.

Put Figure 2 about here.

One may question this conclusion. One might object that the results described above tell something about concept combination only if it can be shown that our beliefs about the properties that are judged to be typical of Oxford graduate students who are carpenters constitute a concept, that is, if they are used in our higher cognitive processes. Otherwise, these results would not bear on the creation of complex concepts. Now, several phenomena suggest that these beliefs are indeed used by our cognitive processes. Particularly, subjects are able to evaluate the typicality of (described) individuals qua 
Oxford graduate students who are carpenters (e.g., Medin and Shoben 1988). This suggests that people use their expectations about which properties are typical of Oxford graduate students who are carpenters to evaluate the typicality of individuals. One might however object that these typicality judgments may be a function of the typicality of these individuals qua Oxford graduate students and of their typicality qua carpenters (Huttenlocher and Hedges 1994). Hence, these judgments may not rely on complex concepts that represent typical properties. However, the typicality of items with respect to a complex concept (OXFORD GRADUATE STUDENT WHO IS A CARPENTER) is often not a function of their typicality with respect to the combined concepts (OXFORD GRADUATE STUDENT and CARPENTER). This happens only when the membership in one category (Oxford graduate students) is believed to be independent from the membership in the other (carpenters) (Hampton 1987, 57; Huttenlocher and Hedges 1994). Hence, the best explanation of these typicality judgments is that subjects do produce a representation of the typical properties of the complex concept and use this representation to make typicality judgments (for other phenomena, see Hampton 1982, 1987, 1988, 1996).

Second, the fact that subjects are able to list the typical properties of Oxford graduate students and of carpenters does not show that their concepts of Oxford graduate students and of carpenters store some information about those properties. For, knowledge of typical properties may not be part of their concepts; instead, it could merely be a piece of background knowledge. However, knowledge of typical properties is used in most, if not all, our cognitive processes (e.g., Rosch and Mervis 1975 on categorization; Osherson 
et al. 1990 on induction; Sloman 1998 on deduction). Thus, the data structures that encode this knowledge are poised for being used in various higher cognitive processes. Hence, they are concepts.

\subsection{Theories and Property Inheritance}

The story is however more complex, for psychologists have shown that the typicality of a property is not the only factor that determines whether it is represented by the complex concept. Property inheritance is also influenced by our theoretical knowledge about the categories that are combined (Medin and Shoben 1988; Murphy 1988, 1990; Rips 1995; Johnson and Keil 2000).

Concept combination requires indeed some modal information about the properties of the original categories, for example about the properties of grandmothers and of spies. Which properties are represented by the complex concept, GRANDMOTHER SPY, is a function of their modal force. Studies show that if the input concepts represent impossible properties (properties typically possessed by the members of one category, say male for spies, but that are believed to be impossible for the members of the other category, say grandmothers), these are never represented by the complex concept; if the input concepts represent necessary properties (properties that are believed to be necessarily possessed by the members of one category, say being the mother of a parent for grandmothers), these are always represented by the complex concept (Hampton 1987).

Moreover, concept combination requires some causal information about the properties of the original categories (Medin and Shoben 1988; Murphy 1988, 1990; 
Johnson and Keil 2000). So-called central properties are preferentially represented by complex concepts. A property is central when it explains causally the possession of other properties. For example, being curved is a central property of boomerangs because subjects believe that boomerangs come back because they are curved. Being made out of wood is less central because it explains few properties of boomerangs. ${ }^{16}$

These results show that during concept combination, we access some modal and causal knowledge. Now, prototypes do not represent any modal nor any causal knowledge, but only some statistical knowledge. Consequently, during concept combination, we access some knowledge that is not represented by prototypes. Now, theories are precisely supposed to represent this form of knowledge. Does this show that to account for concept combination, we need to posit a second type of concepts, i.e., theories, beside prototypes? Not so easily, however. For, one could object that the evidence does not show that we have some theoretical concepts, that is, bodies of theoretical knowledge that are used by default in a large number of cognitive tasks. We may simply rely on some background modal and causal knowledge about the categories that are combined. This is indeed how Hampton himself thinks of the use of modal knowledge in concept combination (Hampton 1997). Being a prototype theorist, he believes that modal knowledge is specifically extracted for the purpose of building complex concepts. Hence, modal knowledge is not used by default in our cognitive processes. I disagree with this interpretation of the evidence. For, we routinely use this very same causal and modal knowledge in other higher cognitive processes (see Keil 1989 on categorization; Coley et al. 1999 on inductive reasoning). The 
theoretical knowledge that is used in concept combination is poised for being used in many cognitive processes. Thus, the data structures that encode this knowledge are concepts.

\subsection{Emergent Properties, Exemplars and Theories}

Not all properties that are represented by a complex concept are represented by the original prototypes. These properties are said to be emergent and this phenomenon is known as property emergence (Hampton 1987, 1997; Kunda et al. 1990; Johnson and Keil 2000; Costello and Keane 2000). For example, Oxford graduate students who are carpenters may be judged to have an artistic character, while neither Oxford graduate students nor carpenters are judged to have an artistic character (Kunda et al. 1990). Several explanations of this phenomenon have been suggested. They are not exclusive. For the sake of argument, I focus on the explanation that relies on exemplars.

Some properties derive from our knowledge of specific members of the complex category (Hampton 1987; Medin and Shoben 1988, 183 sq.). For example, if a subject has to produce on the fly a complex concept like PRESIDENT FROM TEXAS, she may look in her long-term memory for someone who is president and who comes from Texas. Plausibly, she would retrieve the singular representation of G. W. Bush and she would transmit to the complex concept the properties that are represented by this singular memory. She would thus represent presidents from Texas as being right-wing. Hence, concept combination accesses some singular representations. This suggests that these may be poised to be used in our higher cognitive processes, that is, that they are concepts. 


\subsection{Consequences}

Empirical evidence suggests that concept combination rests on three kinds of knowledge: some statistical knowledge about the typical properties of the relevant categories, some causal and modal knowledge about these categories and some knowledge about some members of these categories. Prototypes, theories and exemplars are respectively supposed to encode these kinds of knowledge. Since other cognitive processes rely also on these bodies of knowledge, they qualify as concepts. This is evidence for premise 3 of the argument from explanatory necessity: in order to explain concept combination, we need to assume that prototypes, exemplars and theories exist.

\subsection{Objections}

For the sake of space, I cannot deal at length with the possible objections. For the time being, I merely mention the most prominent ones and sketch some answers.

- Concepts are prototypes - exemplars and theories are merely part of our background knowledge (mutatis mutandis, for exemplars and theories).

Prototype theorists do not deny the existence of exemplars and theories (mutatis mutandis, for exemplar and theory theorists). However, they claim that they are not concepts, plausibly because they are not used by default in our cognitive processes. I have dealt with this objection earlier. In brief, exemplars and theories qualify as concepts alongside prototypes, because they are retrieved by default from our long-term memory to solve a large number of tasks. 
- Do we really need to posit both exemplars and prototypes? In the categorization literature, most psychologists believe that to account for the data, exemplars (Zaki and Nosofsky 2004) or prototypes that represent property correlations and category variability (Barsalou 1990; Hampton 2001) are sufficient.

However, prototype theorists deny that exemplars account for all the categorization data, and vice-versa (e.g., Smith and Minda 2002; Zaki and Nosofsky 2004). To account for the data about human categorization, including neuroimagery data (e.g., Smith et al. 1998) and the performances of amnesic patients (Knowlton 1999; but see Zaki and Nosofsky 2004), one has plausibly to posit both prototypes and exemplars. Moreover, if one takes into consideration other cognitive competences, the need for both types of concepts is even more pressing. For instance, singular representations are often used in reasoning (see the study of case-based reasoning, e.g., Hammond 1989) alongside prototypes (e.g., Osherson et al. 1990) and theories (e.g., Wilson and Keil 2000).

- Theories, exemplars and prototypes have much in common: they denote categories, they store some knowledge about them, they are used in induction, in categorization, they are acquired through experience etc. Hence, the class of concepts is a natural kind. Members of a natural kind have many properties in common beside those properties that are used to identify the kind. This is not the case of concepts. Most of the properties mentioned in the objection are used to identify the class of concepts. Moreover, psychologists are not interested in those properties. What psychologists want to find out is not that concepts store some knowledge about categories, but what kind of knowledge is stored. Similarly, the fact that concepts can be used in induction (or in categorization, or 
learned through experience...) is not a generalization that is of much interest for psychologists. What they want to know is how they are used to solve inductive tasks, to categorize or how they are learned. Now, I have aimed at showing that the class of concepts is not adequate for this purpose. Exemplars, prototypes and theories do not store the same type of knowledge; they are not used in the same kind of inductive process etc. That is, the class of concepts does not support the kind of generalizations psychologists are interested in. It is not a natural kind.

- The evidence shows in fact that each concept is constituted by three elements, or parts - a prototype, a set of exemplars and a theory, not that each category is represented by several concepts.

A similar idea was developed under the name "dual theories of concepts" at the end of the seventies (e.g., Osherson and Smith 1981). More recently, some psychologists have developed new hybrid theories of concepts (e.g., Gelman 2004). Spelling out these views and evaluating them would require a lengthy treatment. In brief, I claim that most of them are terminological variants of the heterogeneity hypothesis, for psychologists are interested in the properties of the parts. To the extent that they differ from the heterogeneity hypothesis, for instance by insisting that one part is more central than the others, I claim that little evidence supports them.

\section{Conclusion}

Most psychologists of concepts and of the higher cognitive functions (categorization, induction...) - particularly, prototype theorists, exemplar theorists and theory theorists - as 
well as some philosophers endorse the received view: despite differences between kinds of concepts, many scientifically interesting properties are true of most, if not all, concepts. Thus, the class of concepts is supposed to be central for a scientific psychology: it is the proper grain to formulate inductive generalizations about the data structures that are used by default in our higher cognitive processes. In brief, it is a natural kind (the NK assumption).

I have aimed at rebutting the NK assumption. I propose instead that the class of concepts divides into kinds that have little in common (the heterogeneity hypothesis). It may be useful to reformulate the argument. The three main psychological theories of concepts posit three kinds of entities, exemplars, theories and prototypes. These theoretical constructs do not share a large set of relevant properties: they encode different kinds of knowledge and are used in different kinds of cognitive processes (Section 4). Now, the research on an important cognitive process, concept combination, suggests that these three theoretical constructs have to be received in our ontology (Section 5). A careful study of the empirical literature on concepts shows that this is true of the other higher cognitive processes. Hence, the data structures that are used by default in our higher cognitive processes have little in common. If concepts are these mental representations, the class of concepts encompasses several kinds of mental representations that have little in common (the argument from explanatory necessity). Hence, even on the broadest notion of natural kind, concepts are not a natural kind.

The implications of this conclusion are far-reaching. Although for the sake of space, I cannot elaborate here, I present the most important ones. First, the notion of 
concept is ill-suited to formulate scientifically relevant generalizations about the mind. Psychologists should focus instead on other classes of mental representations, particularly prototypes, exemplars, and theories (and eventually others). In other words, the notion of concept does not carve the mind at its joints. Second, the controversy between the main psychological theories of concepts is deeply misguided. Concepts are neither prototypes, nor exemplars, nor theories. Some concepts are prototypes, some concepts are sets of exemplars, some concepts are theories. The theory view of concepts, the prototype view of concepts and the exemplar view of concepts are not inconsistent theories about our concepts: instead, they characterize the main features of three basic different kinds of representations. Finally, this position raises a provocative question: if the notion of concept is ill-suited for scientific purposes, do we need it at all? But this is certainly a topic for another day. 
Figures

See the 2 joined documents. 


\section{REFERENCES}

Ashby, F. Gregory, Leola A. Alfonso-Reese, And U. Turken, and Elliott M. Waldron (1998), “A Neuropsychological Theory of Multiple Systems in Category Learning”, Psychological Review 105: 442-481.

Barsalou, Lawrence W. (1990), “On the Indistinguishability of Exemplar Memory and Abstraction in Category Representation”, in Thomas K. Srull and Robert S. Wyer (eds.), Advances in Social Cognition, Vol. III, Content and Process Specificity in the Effects of Prior Experiences. Hillsdale, NJ: Erlbaum, 61-88.

_ (1999), “Perceptual Symbol Systems”, Behavioral and Brain Sciences 22: 577660.

Boyd, Richard (1990), "What Realism Implies and What it does Not”, Dialectica 43: 5-29.

_ (1991), “Realism, Anti-foundationalism and the Enthusiasm for Natural Kinds”, Philosophical Studies 61(1-2): 127-148.

Carey, Susan (1985), Conceptual Change in Childhood. Cambridge, MA: MIT Press.

— (2000), “The Origin of Concepts”, Journal of Cognition and Development 1: 3741.

Coley, John D., Douglas L. Medin, Julia Beth Proffitt, Elizabeth B. Lynch, and Scott Atran (1999), “Inductive Reasoning in Folkbiological Thought”, in Douglas L. Medin and Scott Atran (eds.), Folkbiology. Cambridge, MA: Bradford, 205-232.

Collier, John (1996), “On the Necessity of Natural Kinds”, in Peter Riggs (ed.), Natural Kinds, Laws of Nature and Scientific Reasoning. Dordrecht: Kluwer, 1-10.

Costello, Fintan J., and Mark T. Keane (2000), “Efficient Creativity: Constraint-Guided Conceptual Combination”, Cognitive Science 24: 299-349. 
Fodor, Jerry A. (1974), “Special Sciences”, Synthese 28: 97-115.

(1998), Concepts: Where Cognitive Science Went Wrong. New York: Oxford University Press.

Gelman, Rochel (2004), “Cognitive Development”, in Hal Pashler and Douglas L. Medin (eds.), Stevens’ Handbook of Experimental Psychology, Vol. 2: Memory and Cognitive Processes, Third edition, New York: Wiley.

Goldstone, Robert L., and Alan Kersten (2003), “Concepts and Categorization”, in A. F. Healy and R. W. Proctor (eds.) Comprehensive Handbook of Psychology, Volume 4: Experimental Psychology, New Jersey: Wiley, 599-621.

Gopnik, Alison, and Andrew N. Meltzoff (1997), Words, Thoughts, and Theories. Cambridge, MA: MIT Press.

Griffiths, Paul E. (1997), What Emotions Really Are. Chicago: Chicago University Press. Hacking, Ian (1991), “A Tradition of Natural Kinds”, Philosophical Studies 61: 109-126. Hahn, Ulrike, and Nick Chater (1998), “Similarity and Rules: Distinct? Exhaustive? Empirically Distinguishable?”, Cognition 65: 197-230.

Hammond, Kristian J. (1989), Case-based Planning: Viewing Planning as a Memory Task. San Diego, CA: Academic Press.

Hampton, James A. (1982), “A Demonstration of Intransitivity in Natural Categories”, Cognition 12: 151-164.

(1987), “Inheritance of Attributes in Natural Concept Conjunctions”, Memory and Cognition 15: 55-71. 
_ (1988), “Overextension of Conjunctive Concepts: Evidence for a Unitary Model of Concept Typicality and Class Inclusion”, Journal of Experimental Psychology: Learning, Memory, and Cognition 14: 12-32.

— (1996), “Conjunctions of Visually-Based Categories: Overextension and Compensation”, Journal of Experimental Psychology: Learning, Memory and Cognition 22: 378-396.

_ (1997), “Conceptual Combination”, in Koen Lamberts and David Shanks (eds.), Knowledge, Concepts and Categories. Cambridge, MA: MIT Press, 133-160. _ (2001), “The Role of Similarity in Natural Categorization”, in Ulrike Hahn and Michael Ramscar (eds.), Similarity and Categorization. Oxford: Oxford University Press, 13-28.

Hull, David (1978), “A Matter of Individuality”, Philosophy of Science 45: 335-360. Huttenlocher, Janellen, and Larry V. Hedges (1994), “Combining Graded Categories: Membership and Typicality”, Psychological Review 101(1): 157-165.

Johnson, Christine, and Frank C. Keil (2000), “Explanatory Understanding and Conceptual Combination”, in Frank C. Keil and Robert A. Wilson (eds.), 2000, 328-359. Juslin, Peter, and Magnus Persson (2002), “PROBabilities from Exemplars (PROBEX): a “Lazy” Algorithm for Probabilistic Inference from Generic Knowledge”, Cognitive Science 26: 563-607.

Keil, Frank C. (1989), Concepts, Kinds, and Cognitive Development. Cambridge, MA: MIT Press. 
Keil, Frank C., and Robert A. Wilson (eds.) (2000), Explanation and Cognition.

Cambridge, MA: MIT Press.

Knowlton, Barbara J. (1999), "What can Neuropsychology Tell us about Category

Learning”, Trends in Cognitive Science 3(4): 123-124.

Kunda, Ziva, Dale T. Miller, and Theresa Claire (1990), “Combining Social Concepts: The Role of Causal Reasoning”, Cognitive Science 14: 551-577.

Laurence, Stephen, and Eric Margolis (1999), “Concepts and Cognitive Science”, in Stephen Laurence and Eric Margolis (eds.), Concepts, Core Readings. Cambridge, MA: MIT Press, 3-81.

Machery, Edouard (ms), “The Birth of the Psychology of Concepts in the Nineteenth Century”.

Margolis, Eric (1994), “A Reassessment of the Shift from the Classical Theory of Concepts to Prototype Theory”, Cognition 51(1): 73-89.

Medin, Douglas L., and M. M. Shaffer (1978), “Context Theory of Classification Learning”, Psychological Review 85: 207-238.

Medin, Douglas L., and Edward J. Shoben (1988), “Context and Structure in Conceptual Combination”, Cognitive Psychology 20: 158-190.

Medin, Douglas L., Elisabeth B. Lynch, and Karen O. Solomon (2000), “Are there Kinds of Concepts?”, Annual Review of Psychology 51: 121-147.

Mill, John Stuart (1905), A System of Logic. London: Routledge.

Murphy, Gregory L. (1988), “Comprehending Complex Concepts”, Cognitive Science 12: 529-562. 
_ (1990), “Noun-Phrase Interpretation and Conceptual Combination”, Journal of Memory and Language 29: 259-288.

— (2002), The Big Book of Concepts. Cambridge, MA: MIT Press.

Murphy, Gregory L., and Douglas L. Medin (1985), “The Role of Theories in Conceptual Coherence”, Psychological Review 92: 289-316.

Nosofsky, Robert M. (1988), “Exemplar-Based Accounts of Relations Between Classification, Recognition, and Typicality”, Journal of Experimental Psychology: Learning, Memory and Cognition 14: 700-708.

Osherson, Daniel N., and Edward E. Smith (1981), “On the Adequacy of Prototype Theory as a Theory of Concepts”, Cognition 9: 35-58.

Osherson, Daniel N., Edward E. Smith, O. Wilkie, A. Lopez, and Eldar Shafir (1990), “Category-Based Induction”, Psychological review 97: 185-200.

Prinz, Jesse J. (2002), Furnishing the Mind: Concepts and their Perceptual Basis. Cambridge, MA: MIT Press.

Putnam, Hilary (1975), “The Meaning of 'Meaning””, in Hilary Putnam, Mind, Language, and Reality. Cambridge: Cambridge University Press, 215-271.

Quine, Willard V. O. (1969), “Natural Kinds”, in William V. O. Quine, Ontological Relativity and Other Essays. New York: Columbia University Press.

Rips, Lance J. (1995), “The Current Status of the Research on Concept Combination”. Mind and Language 10: 72-104.

Rosch, Eleanor, and Carolyn B. Mervis (1975), “Family Resemblance: Studies in the Internal Structure of Categories”, Cognitive Psychology 7: 573-605. 
Sloman, Steven A. (1998), “Categorical Inference is Not a Tree: The Myth of Inheritance Hierarchies”, Cognitive Psychology 35: 1-33.

Smith, Edward E., and Daniel N. Osherson (1989), "Similarity and Decision Making”, in Stella Vosniadou and Andrew Ortony (eds.), Similarity and Analogical Reasoning. Cambridge: Cambridge University Press, 60-75.

Smith, Edward E., Christopher Langston, and Richard E. Nisbett (1992), “The Case for Rule in Reasoning”, Cognitive Science 16: 1-40.

Smith, Edward E., and Douglas L. Medin (1981), Categories and Concepts. Cambridge, MA: Harvard University Press.

Smith, Edward E., Daniel N. Osherson, Lance J. Rips, and Margaret Keane (1988), “Combining Prototypes: A Selective Modification Model” Cognitive Science 12: 485-521.

Smith, Edward E., Andrea L. Patalano, and John Jonides (1998), “Alternative Strategies of Categorization”, Cognition 65: 167-196.

Smith, J. David (2002), “Exemplar Theory’s Predicted Typicality Gradient can be Tested and Disconfirmed”, Psychological Science 13: 437-442.

Smith, J. David, and John Paul Minda (2002), “Distinguishing Prototype-Based and Exemplar-Based Processes in Category Learning”, Journal of Experimental Psychology: Learning, Memory, and Cognition 28: 800-811.

Zaki, Safa R., and Robert M. Nosofsky (2004), “False Prototype Enhancement Effects in Dot Pattern Categorization” Memory \& Cognition 32(3): 390-398. 
Figure Titles

Figure 1: The Heterogeneity Hypothesis

Figure 2: Property Inheritance 


\section{FOOTNOTES}

${ }^{1}$ In what follows, I stick to this psychological use of the term “concept”.
${ }^{2}$ For reviews, see Smith and Medin 1981; Lambert and Shanks 1997; Murphy 2002;

Goldstone and Kersten 2003. In philosophy of psychology, see Margolis 1994; Laurence and Margolis 1999; Prinz 2002.

${ }^{3}$ There are other views of concepts (e.g., Barsalou 1999; Prinz 2002). However, the argument is strengthened if we have to admit a fourth basic kind of mental representations (provided that it has little in common with the other basic kinds).

${ }^{4}$ The term "prototype" is used ambiguously to design the best member of a category and the representation of a category. I use the term in this second sense.

${ }^{5}$ One might object that this account assumes paradoxically that a prototype contains the very concept it is supposed to constitute. The appeal of this mereological objection is however illusory. For the sake of argument, suppose that the classical view of concepts is true. The concept BACHELOR is a data structure made out of three elements, namely, the beliefs that all bachelors are male, that they are all adult and that none is married. Since the identity of each element is conditional on the presence of the two others, it is not the case that the concept contains the very concept it is supposed to constitute. Taken separately, each element does not contain the concept BACHELOR.

${ }^{6}$ In the literature, exemplars are taken to represent either individuals or specific encounters with individuals. In the first case, human beings have one representation for each (represented) member of a category. I have one exemplar of Fido, another one of Spot... In 
the second case, whenever I meet Fido, I produce a new exemplar (Nosofsky 1988). I favor the first idea.

${ }^{7}$ This idea is often put very loosely in the empirical literature. One finds usually two ideas: (i) concepts are enmeshed within theories and (ii) concepts are theories. I am interested in the second claim.

${ }^{8}$ Other properties, for example neural properties, may turn out to be important to characterize representations. Then, we would have to reevaluate the denial of the NK assumption.

${ }^{9}$ Some prototype theories are more complex.

${ }^{10}$ If we endorse the heterogeneity hypothesis, we have to assume that there are several processes to categorize, several processes to reason inductively etc. This raises an exciting, but yet understudied question: how do these cognitive processes interact or compete?

${ }^{11}$ How psychologists’ empirical work on concept combination relates to the compositionality arguments in philosophy of mind (Fodor 1998) is an important topic that awaits further research (but see Prinz 2002).

12 There are several kinds of concept combination. “Oxford graduate student that is a carpenter” (Hampton 1987) and "red bicycle” (Smith et al. 1988) expresses arguably two different types of complex concepts.

${ }^{13}$ Because complex expressions like “red wine” are common, they may express concepts that are permanently stored in long-term memory, that is, simple concepts (Rips 1995). ${ }^{14}$ Notice that I am not claiming that complex concepts are prototypes. Although complex concepts do encode some information about the properties that are judged to be typical, as 
do prototypes, they may also encode some modal and some causal information - see

below.

${ }^{15}$ See below.

${ }^{16}$ In the literature, it is unclear how the complex concept represents the causal and the modal knowledge. What is clear is that these two types of knowledge affect the creation of complex concepts. 